Original article

\title{
Diagnostic comparison of biochemical profile in patients with Covid-19, dengue and Acute Febrile illness: Implications for patient management
}

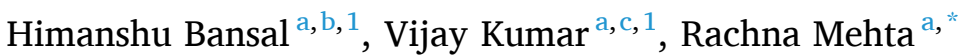 \\ ${ }^{a}$ Amity Institute of Neuropsychology \& Neurosciences, Amity University, Noida, Uttar Pradesh, India \\ ${ }^{\mathrm{b}}$ Department of Health and Family Welfare, Civil Hospital Narwana, Haryana, India \\ ${ }^{c}$ Department of Audiology \& Speech-Language Pathology, Amity University, Gurgaon, Haryana, India
}

\section{A R T I C L E I N F O}

\section{Keywords:}

Covid-19

Comorbid condition

Dengue

\begin{abstract}
A B S T R A C T
Purpose: Although there are specific laboratory tests available for the diagnosis of Covid-19 and dengue, during the present pandemic era of prioritized focus on Covid-19 assessment, there are possibilities that persons with dengue may remain undiagnosed. The present study explores the role of biochemical markers in the differential diagnosis of Covid-19 and dengue.

Methods: A total of 212 participants with Acute Febrile Illness were tested for Covid-19 and dengue at the secondary care hospital, Civil Hospital Narwana, Haryana, India. The Covid-19 and dengue diagnosis were performed using standard tests followed by hematological profiling which included neutrophil lymphocyte ratio (NLR), platelet count, Vitamin D3 assessment, SGOT, SGPT, and SPO2 concentration levels.

Results: Out of 212 participants, 118 were diagnosed with Covid-19 positive only, 18 dengue positive only, 5 coinfected with Covid-19 and dengue, and 71 persons with Acute Febrile Illness (control group). ANOVA revealed that mean SPO2 was significantly lower in Covid-19 and dengue than control, while SGPT and SGOT levels of Covid-19 and dengue patients were significantly higher than the control group. The mean NLR was significantly higher in Covid-19 and dengue than control and Vitamin D3 levels were significantly reduced for Covid-19 patients. Besides, thrombocytopenia was observed only in dengue patients.

Conclusion: The results advocate the potential use of combinations of these makers in differential diagnosis of these two fatal viral conditions and can help by enabling the adaptation of the therapeutic conduct to the needs of individual patients.
\end{abstract}

\section{Introduction}

The episodes of co-infection of Covid-19 and dengue have been reported in Asian countries especially in Indonesia, Pakistan and Singapore and in South America during pandemic spread. ${ }^{1-3}$ In the tropical countries, where dengue is an epidemic, incidence of Covid-19 has further increased the health challenges due to the co-circulation of these causative viruses. ${ }^{4-6}$ The exact severity of co-infection of Covid-19 and dengue is yet not completely understood, and thus requires considerable attention, as more than one type of pathogen in the body may manifest unpredictable outcomes posing a serious health threat which can be fatal. The evidences from the past experience suggest that co-epidemic of flu and dengue, or malaria and dengue further increased the severity of the existing disease especially in the tropical regions. ${ }^{7,8}$
The clinical diagnosis of these diseases during co-infection especially in rural and semi-urban areas where the diagnostic and laboratory facilities are very limited, poses a big challenge.

A similar trend of co-occurrence of two fatal viruses of Covid-19 and dengue has come into picture in a tropical country like India. ${ }^{9,10}$ The risk of incidence of co-infection is higher during the rainy season as mid-June to mid-September is the suitable time for community spread of dengue virus in India. Individuals with underlying chronic conditions such as diabetes, tuberculosis, cancer, heart diseases etc. are more prone to have severe health complications caused by the co-infection with the fatal viral infections. ${ }^{11}$ Especially for those individuals who are either being home isolated (Covid-19 positive) or being home quarantined (Covid-19 suspected) as appropriate tests for complete blood count, liver enzyme test, electrolytes balance etc. are not being done appropriately for each individual attributing to logistic limitations.

\footnotetext{
* Corresponding author.

E-mail address: rmehta2@amity.edu (R. Mehta).

1 Equal Authorship.
} 


\section{Abbreviations used}

\section{CBC Complete Blood Analysis}

Covid-19 Corona Virus Disease 2019

ICMR Indian Council of Medical Research

ILI Influenza like Illness

NLR Neutrophil lymphocyte ratio

NS1 Ag Test Non-Structural Protein 1 Antigen

LFT Liver Function Test

RAT Rapid Antigen Test

RT-PCR Reverse Transcriptase- Polymerase Chain Reaction

SGOT serum glutamic-oxaloacetic transaminase

SGPT serum glutamic-pyruvic transaminase

SPO2 Saturation of Peripheral Oxygen

The biggest challenge in this scenario is simultaneous combat of several other epidemic diseases such as acute febrile illness or Influenza like illness symptoms along with dengue. Accurate and timely differential diagnosis across these two virus vector-borne diseases will help in reducing the fatality rate and in designing better healthcare facilities. In the present study, assessment of serological markers and other biochemical changes were deciphered in patients suffering from Covid19 or dengue or both. In the present scenario, it is utmost essential that patients with severe illness must be hospitalized and all necessary tests must be performed to reduce the chances of mortality. Although telemedicine and indirect consultations have facilitated patient monitoring and has provided immediate medical help, however, it is not preferable in cases of coinfection.

\section{Materials and methods}

Participants: A total of 212 participants with Acute Febrile Illness/ Influenza like Illness were investigated and tested for Covid-19 and dengue based on symptoms. Out of 212 participants, 118 were diagnosed with Covid-19 positive only, 18 dengue positive only, 5 coinfected with Covid-19 and dengue, and 71 persons with Acute Febrile Illness (control group). All the participants were screened from all the units of a secondary care hospital namely Civil Hospital Narwana, Haryana, India. The written informed consent was obtained from all the participants with Acute Febrile Illness/Influenza like Illness and only who agreed to voluntarily participate in this study was included. No prestudy criteria for age and gender were set, as we wanted to retain the variability of the population based on the hypothesis of this study. The participants without any Acute Febrile Illness/Influenza like Illness or asymptomatic were also excluded.

Covid-19 testing procedure: The nasopharyngeal swabs were taken for each participant for Rapid Antigen Test (RAT) and Reverse Transcriptase- Polymerase Chain Reaction (RT-PCR) tests for the detection of Covid-19 virus. The RAT was done by using the kits provided under the Government of India facility known as Standard Q Covid-19 Ag kit, which comes with an inbuilt Covid-19 antigen test device, viral extraction tube with viral lysis buffer and sterile swab for sample collection. A swab from the nose was collected for this test, where there is a high likelihood of virus particles being present. The swab was then dipped in a solution which contains viral lysis buffer which inactivates the virus, and then transferred to a test strip. The test strip houses antibodies that bind to coronavirus proteins and hold them in place as the fluid spreads. The sample was considered positive for coronavirus when coloured lines showed up on the paper strip in 15-20 min. The symptomatic individuals who tested negative for Covid-19 by rapid antigen test were tested sequentially by RT-PCR to rule out infection, whereas a positive test is considered as a true positive and does not need reconfirmation by RT-PCR test.
Haematological Profile: Complete blood analysis (CBC), and Liver Function Test (LFT) was done by drawing $5 \mathrm{ml}$ blood sample of each participant. Values of Platelets $<1,40,000 /$ microL, neutrophil lymphocyte ratio (NLR) $<4$, saturation of peripheral oxygen (SPO2) $<92 \%$, Sodium $<130 \mathrm{mEq} / \mathrm{L}$ were considered as decreased while values of serum glutamic pyruvic transaminase (SGPT) $>50$ and serum glutamicoxaloacetic transaminase (SPOT) $>45$ units per liter of serum were considered as raised. $\mathrm{CBC}$ was repeated in 42 participants, who were complaining of retro-orbital pain, severe body aches, cough and fever. The NS1 Ag test was done, for detection of dengue virus with the help of commercially available Dengue NS1 Rapid dip Insta Serum Test kits, in the patients with $<80,000$ platelets $/ \mu$ l which was found using repeated blood sampling for CBC. The dengue NS1 Rapid Test is a qualitative, membrane based immunoassay for the detection of NS1 antigen in human serum. The rapid test membrane is pre-coated with a NS1 specific antibody on the test line region and utilizes a separate control to assure assay flow and performance. During testing, the test sample was added directly to the sample region and the test was placed into a well containing 3 drops of buffer. The buffer and serum mix and interact with NS1-specific monoclonal antibodies conjugated to gold nanoparticles. The solution migrates upward on the membrane to react with the antiNS1 antibody on the membrane. If NS1 antigen is present, a red line will appear at the test line, which was then considered as reactive. The SPO2 levels were checked using pulseoximeter. Pulse oximeter consists of a computerized monitor and a probe. The probe was attached to the patient's finger tip of the hand and the monitor displayed the reading of how saturated the patient's blood is with oxygen. Vitamin D3 level was measured for 38 patients with Covid-19 and 19 patients with dengue positive. For vitamin D3 estimation, a small amount of blood was collected from the vein into a test tube or vial. Blood was analysed for the quantitative determination of 25-hydroxyvitamin D using in vitro diagnostic with LUMIPULSE® G System. The 25-hydroxyvitamin D is the major form found in the blood and is the relatively inactive precursor to the active hormone, 1,25-dihydroxyvitamin D. Levels of vitamin D found above $30 \mathrm{ng} / \mathrm{ml}$ were considered as optimum levels while below $30 \mathrm{ng} / \mathrm{ml}$ was insufficient and less than $20 \mathrm{ng} / \mathrm{ml}$ was considered as a deficient condition.

Data analysis: The Shapiro-Wilk test was performed, and it was observed that the data obtained for SPO2, SGPT, SGOT, platelet count and the NLR were approximately normally distributed. Level of significance was tested at $\mathrm{p}<0.05$ for all the results of this study and expressed as \pm SEM. All statistical analysis was performed using the SPSS statistical ${ }^{\circledR}$ package, version 20.0 (SPSS inc., Chicago, IL, USA) for Windows ${ }^{\circledR}$.

\section{Results}

Out of the 212 participants, 118 were Covid-19 positive only, 18 dengue only, 5 were co-infected with both Covid-19 and dengue, and 71 were neither Covid-19 nor dengue positive but had Acute Febrile Illness symptoms. Based on the occurrence of symptoms and as the patients reported to the healthcare center, the gender wise distribution is hereby provided; for Covid-19 group ( $n=118$ ): 72 males and 46 females; for dengue group $(\mathrm{n}=18)$ : 10 males and 8 females; and or control group ( = 71): 47 males and 24 females. The NS1Ag test was administered on those patients whose platelet count was less than 80000. It was observed that 8 out of 123 Covid-19 positive cases had platelet count less than 80000 with mean platelet count of $55125 \pm 13108$. Out of these 8,5 cases whose platelet count was below 80000 were tested positive for dengue, i.e. 5 cases out of 123 Covid-19 positive cases had co-infection of dengue as well. While doing statistical analysis, the 5 cases of coinfection of Covid-19 and dengue were neither included in Covid-19 nor in dengue groups.

The obtained data were analysed to identify whether various serological markers differs across two viral conditions such as Covid-19 and dengue, the SPO2, SGPT, SGOT, platelet count and NLR were performed 
for each participant who were tested positive for RAT/RT-PCR (Covid19 confirmed cases) and NS1 Ag confirmed test (dengue positive cases). Mean SPO2, SGPT, SGOT, platelet count and NLR were obtained across the Covid-19 and dengue conditions. Attributing to the approximate value of SPO2, SGPT and SGOT out of 100, these three dependent and continuous variables were compared together across the two virus infected groups while the mean platelet count and mean NLR were compared separately. One-way ANOVA test revealed a significant difference in the values of SPO2 $(\mathrm{F}(2,204)=71.71, \mathrm{p}<0.001), \operatorname{SGPT}(\mathrm{F}(2$, $204)=74.58, \mathrm{p}<0.001)$, and SGOT $(\mathrm{F}(2,204)=162.49, \mathrm{p}<0.001)$ across the Covid-19, dengue, and control groups (Fig. 1a). The LSD posthoc test further revealed that the mean SPO2 level for the Covid-19 and the dengue groups were significantly lower $(\mathrm{p}<0.001)$ than the control group, however, the Covid-19 and dengue groups were not significantly different from each other $(\mathrm{p}=0.79)$. Similarly, the mean SGPT level for the Covid-19 and the dengue groups were significantly higher $(\mathrm{p}<$ 0.001) than the control group, however, the Covid-19 and dengue groups did not differ significantly with each other $(\mathrm{p}=0.95)$ (Fig. 1). The mean SGOT level for the Covid-19 and the dengue groups were significantly higher $(\mathrm{p}<0.001)$ than the control group, however, the Covid-19 and dengue conditions did not vary significantly from each other $(\mathrm{p}=0.26)$.

Data were further investigated to understand whether the biochemical markers of patients with co-infection of Covid-19 and dengue were more severe, as evident in symptoms, compared to Covid19 only, dengue only, or control groups. The one-way ANOVA revealed that SGPT and SGOT levels of coinfection group was significantly higher ( $p<0.001$ ) than the control group, while SPO2 was lower than the control group, however no statistical significant difference was observed for co-infection group across the Covid-19 and the dengue groups (Fig. 1b).

Furthermore, the mean platelets count was also compared across the groups i.e. the Covid-19, dengue, and control conditions. One-way ANOVA findings revealed that the mean platelets count across the three groups $(\mathrm{F}(2,204)=89.59, \mathrm{p}<0.001)$ varied significantly. The LSD Post-hoc test further revealed that the mean platelets count for the dengue group was significantly lower $(p<0.001)$ than the Covid-19 and the control groups (Fig. 2a). The mean platelet count was also compared across the males and female participants across groups and it was observed that for dengue group, the mean platelet count for females was significantly lower than the males $(\mathrm{F}(1,17)=14.94, \mathrm{p}<0.001)$ (Fig. 2a). No significantly difference was observed in the platelet count across gender for Covid-19 and control group.

The mean NLR ratio was also compared across the groups i.e. the Covid-19, dengue, and control conditions. One-way ANOVA findings revealed a significant variation in the mean NLR ratio across the three groups $(\mathrm{F}(2,204)=266.7658, \mathrm{p}<0.001)$. The LSD Post-hoc test further revealed that the mean NLR ratio for the Covid-19 and the dengue groups were significantly higher $(p<0.001)$ than the control group (Fig. 2b). Similarly, the mean NLR ratio for the dengue was significantly higher $(\mathrm{p}<0.05)$ than the Covid-19. No significant difference was observed in the mean NLR ratio across male and female participants of all the three groups (Fig. 2b).

Further, vitamin D3 level was measured across the Covid-19, dengue, and control groups. Levels of vitamin D found above $30 \mathrm{ng} / \mathrm{ml}$ were considered as optimum levels while below $30 \mathrm{ng} / \mathrm{ml}$ was insufficient, and less than $20 \mathrm{ng} / \mathrm{ml}$ was considered as a deficient condition. One-way ANOVA findings revealed that the Vitamin D3 level in persons with Covid-19, dengue and control groups were significantly varying $(\mathrm{F}(2$, $103)=47.16, \mathrm{p}<0.001$ ). (Fig. 3). The Post-hoc analysis revealed that Vitamin D3 level of Covid-19 group was significantly lower $(\mathrm{p}<0.001)$ than the dengue and the control group, besides, no significant difference was observed between the dengue and control group $(\mathrm{p}=0.3)$. Data was further analysed to compare the mean Vitamin D3 level across the gender of three groups. However, no significant difference was observed across gender for the Covid-19 $(\mathrm{F}(1,37)=0.12, \mathrm{p}=0.91)$, dengue groups $(\mathrm{F}(1,8)=0.19, \mathrm{p}=0.74)$, and control groups $(\mathrm{F}(1,58)=0.21, \mathrm{p}$ $=0.69$ )(Fig. 3).

\section{Discussion}

The study depicts the role of various biochemical markers and Vitamin D3 level in diagnostic comparison across the118 patient of Covid-19 only, 18 dengue only, 71 acute febrile illness and the 5 coinfection of Covid-19 and dengue in rural population of Haryana, India. The variable number of participants across each group can be attributed to the occurrence of infection and their reporting to the hospital, where this study was conducted. The confirmation of Covid-19 was done with RAT/RT-PCR test and dengue was confirmed using NS1 Ag test.

The biochemical markers such as SPO2, SGPT and SPOT, mean NLR and platelet count were compared across the Covid-19 only, dengue only, and the control group. Results revealed that mean SPO2 was significantly lower in Covid-19 and dengue than control, while SGPT
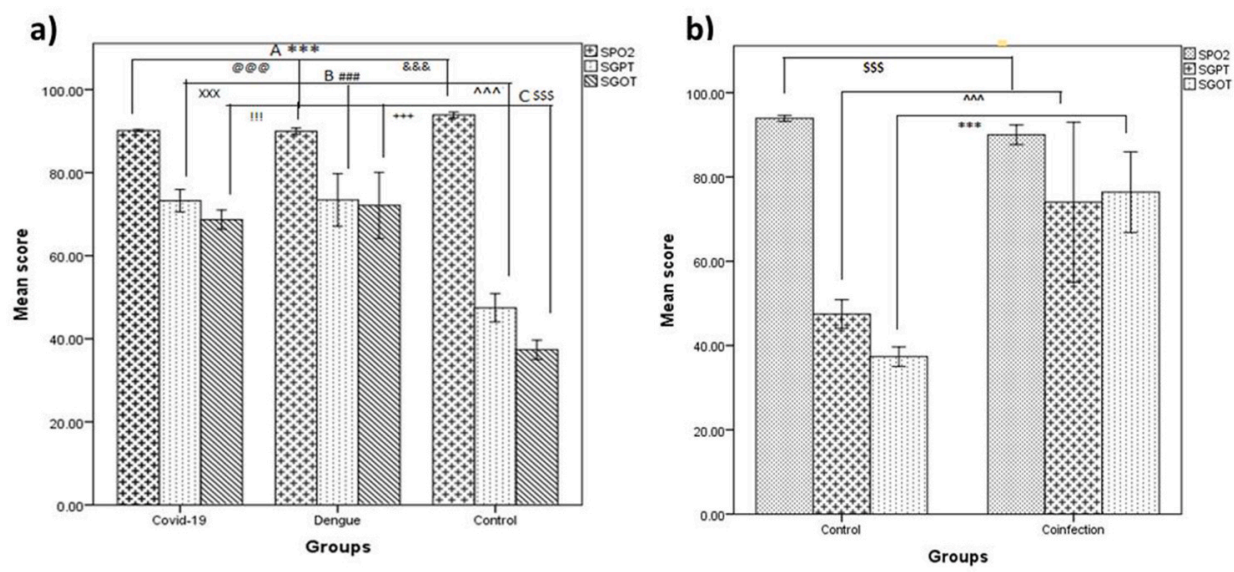

Fig. 1. a): Mean SPO2, SGPT and SGOT score across 118 Covid-19, 18 dengue and 71 control groups are represented through histogram bars. Level of significance was tested at $\mathrm{p}<0.05$. $(* * *)$ - significance of SPO2 at $\mathrm{p}<0.001$ across Covid-19, dengue and control groups, (@@@)significance across Covid-19 and control groups, $(\& \& \&)$ - significance across dengue and control at $\mathrm{p}<0.001,(\# \# \#)$ - significance of SGPT at $\mathrm{p}<$ 0.001 across Covid-19, dengue and control groups, (XXX) - significance across Covid-19 and control, $\left({ }^{\wedge \wedge}\right)$ - significance across dengue and control at $\mathrm{p}<0.001,(\$ \$)$ - level of significance of SGOT at $\mathrm{p}<0.001$ across Covid-19, dengue and control groups, (!!!)- significance across Covid-19 and control, $(+++)$ - significance across dengue and control at $\mathrm{p}<0.001$; Results are shown as mean \pm SEM

Fig. 1b): Mean SPO2, SGPT and SGOT score across 5 co-infection, and 71 control groups are represented through histogram bars. Level of significance was tested at $\mathrm{p}<0.05$. (\$\$)- significance of SPO2 at $\mathrm{p}<0.001$ across Covid-19, dengue, co-infection, and control groups, ( $\left.{ }^{\wedge}\right)$ - significance of SGPT at $\mathrm{p}$ $<0.001$ across Covid-19, dengue, co-infection and control groups, and $(* * *)$-level of significance of SGOT at $\mathrm{p}<0.001$ across Covid-19, dengue, co-infection and control groups; Results are shown as mean \pm SEM. 


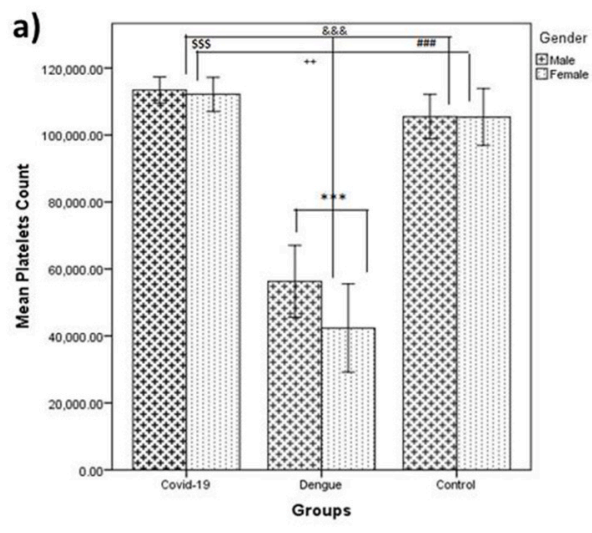

b)

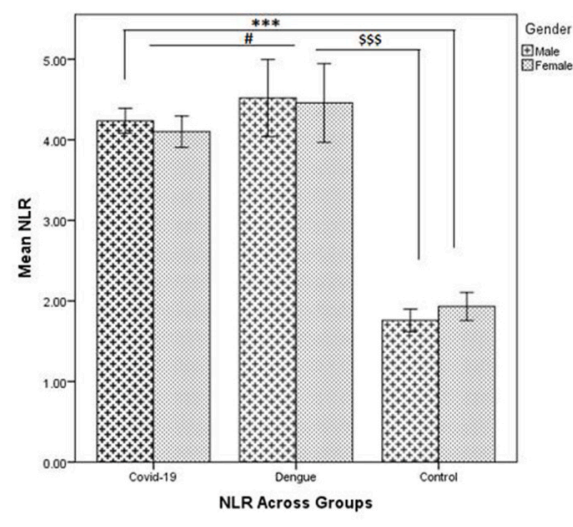

sents level of significance across Covid-19 and dengue groups at $\mathrm{p}<0.05$; Results are shown as mean \pm SEM.
Fig. 2. a): Mean Platelets count across Covid-19, dengue and control groups. Level of significance was tested at $p<0.05$. (\&\&\&) - significance of mean platelets count at $\mathrm{p}<0.001$ across the Covid-19, dengue and control groups, (\$\$)- significance across Covid-19 and dengue, (\#\#\#)- significance across dengue and control, and $(++)$ significance across Covid-19 and control groups at $\mathrm{p}<0.01$, (***)- level of significance of mean platelet count across males and females of dengue group at $\mathrm{p}<0.001$; Results are shown as mean \pm SEM.

Fig. 2b): Mean NLR ratio across Covid-19, dengue and control groups. Level of significance was tested at $\mathrm{p}<0.05$. (***) represents level of significance of mean NLR ratio at $\mathrm{p}<0.001$ across the Covid-19 and control groups, (\$\$) represents level of significance across dengue and control, and (\#) repre-

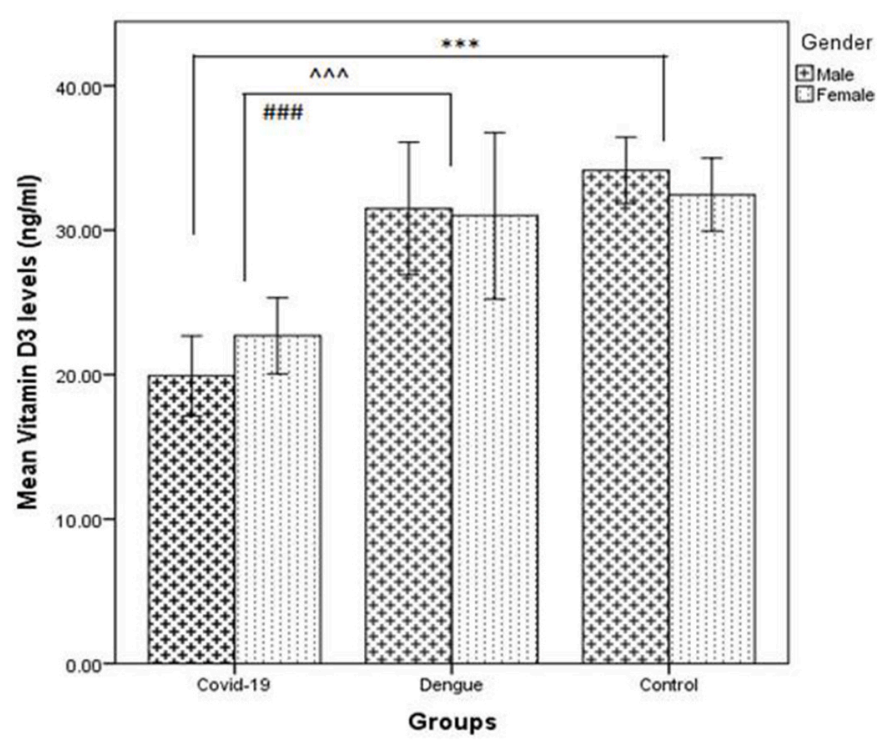

Fig. 3. Mean Vitamin D3 level in (ng/ml) across Covid-19, dengue and control groups and across gender. Level of significance was tested at $\mathrm{p}<0.05$. (***) represents level of significance at $\mathrm{p}<0.001$ across the Covid-19, dengue and control groups; $\left({ }^{\wedge n}\right)$ represents level of significance at $p<0.001$ across the Covid-19 and control groups; and, (\#\#\#) represents level of significance at $\mathrm{p}<$ 0.001 across the Covid-19 and dengue groups. Results are shown as mean \pm SEM.

and SGOT levels of Covid-19 and dengue patients were significantly higher than the control group. The mean NLR of the Covid-19 and dengue were significantly higher than the control group while thrombocytopenia was observed only in dengue patients. We evaluated the diagnostic and prognostic accuracy of the NLR in COVID-19 patients. Moderate and severe COVID-19 infected patients had significantly higher levels of NLR than mild and non-severely infected patients. The biochemical findings of this study are supported by few of the similar studies done earlier. ${ }^{12-14,23}$ In the present pandemic era, where there is prime focus on Covid-19 assessment, its intervention and prevention, there are high chances that co-occurrence of dengue might get ignored by the leading healthcare professionals across regional and national boundary. Although, the number of participants in co-infection group was only five, the preliminary empirical analysis of biochemical markers of co-infection group was compared across Covid-19 only, dengue only and control group only. The SPO2, SGPT, and SPOT of co-infection group was significantly lower than the control group. These outcomes further motivates to extend further research in this direction as previous onset of more than one epidemic suggests that the comorbidity of Covid-19 and dengue can be more fatal, thus, presenting a greater health challenges which needs to be addressed at the policy level by the healthcare providers. ${ }^{11,15,16}$ Vitamin D3 level was compared across the Covid-19 and dengue groups and it was observed that the levels of vitamin D3 level were significantly reduced for Covid-19 positive participants as compared to the dengue group irrespective of gender. Vitamin D has several beneficial effects on the immune system and contributes significantly to the immune system health. ${ }^{17-19}$ The role of vitamin D3 is also crucial in regulating the respiratory infection and thus vitamin D3 supplements play an important role in the prevention of viral infection. ${ }^{20-22}$ Additional investigations like interleukin (IL-6) and high resolution computerized tomography of chest could not be done due to lack of facility in the hospital where data was collected.

The advisory by the Indian Council of Medical Research (ICMR) recommends that TEST, TRACK AND TREAT is the only way to prevent the spread of infection and save lives. Proper testing protocols/strategies for the diagnosis of either of the two fatal diseases or co-infections should be implemented for better prevention, treatment and, hence reducing mortality. There are few distinguishing conditions between these overlapping vector-borne conditions which must be considered critically. Fever in dengue has a characteristic feature called as "Breakbone Fever" which can be a distinguishing factor in comparison with Covid-19 fever. Respiratory distress is more prominent in Covid-19 than in Dengue Fever. While in Covid-19 we usually see leucocytosis, leukopenia is more often in dengue. Rhinorrhoea is generally seen in Covid-19 infection and not in Dengue Fever while hemoglobin levels are generally increased in dengue fever in comparison to Covid-19. Covid19 can present non-febrile patients with or without rhino pharyngitis and cough, here dengue should not be a first-line diagnosis. Therefore, diagnosis of febrile patients should be organized to allow diagnosis of both dengue and Covid-19 without delays due to Covid-19 constraints (Fig. 4). In addition to Covid-19 screening, appropriate tests must be done by the clinicians and the health care providers so that patients of acute febrile illness symptoms should not remain undiagnosed and proper mental health counseling could be administered to improve the mental health of the patients suffering from any of the fatal viral infections. Regarding vector control, health promotion should encourage populations to look out for potential vector breeding places and protect themselves from mosquito bites. Local authorities should be very vigilant and activate strategic services essential to vector control (waste management, maintenance of public spaces, intra-domiciliary interventions, notably around cases). Regarding Covid-19, testing of 


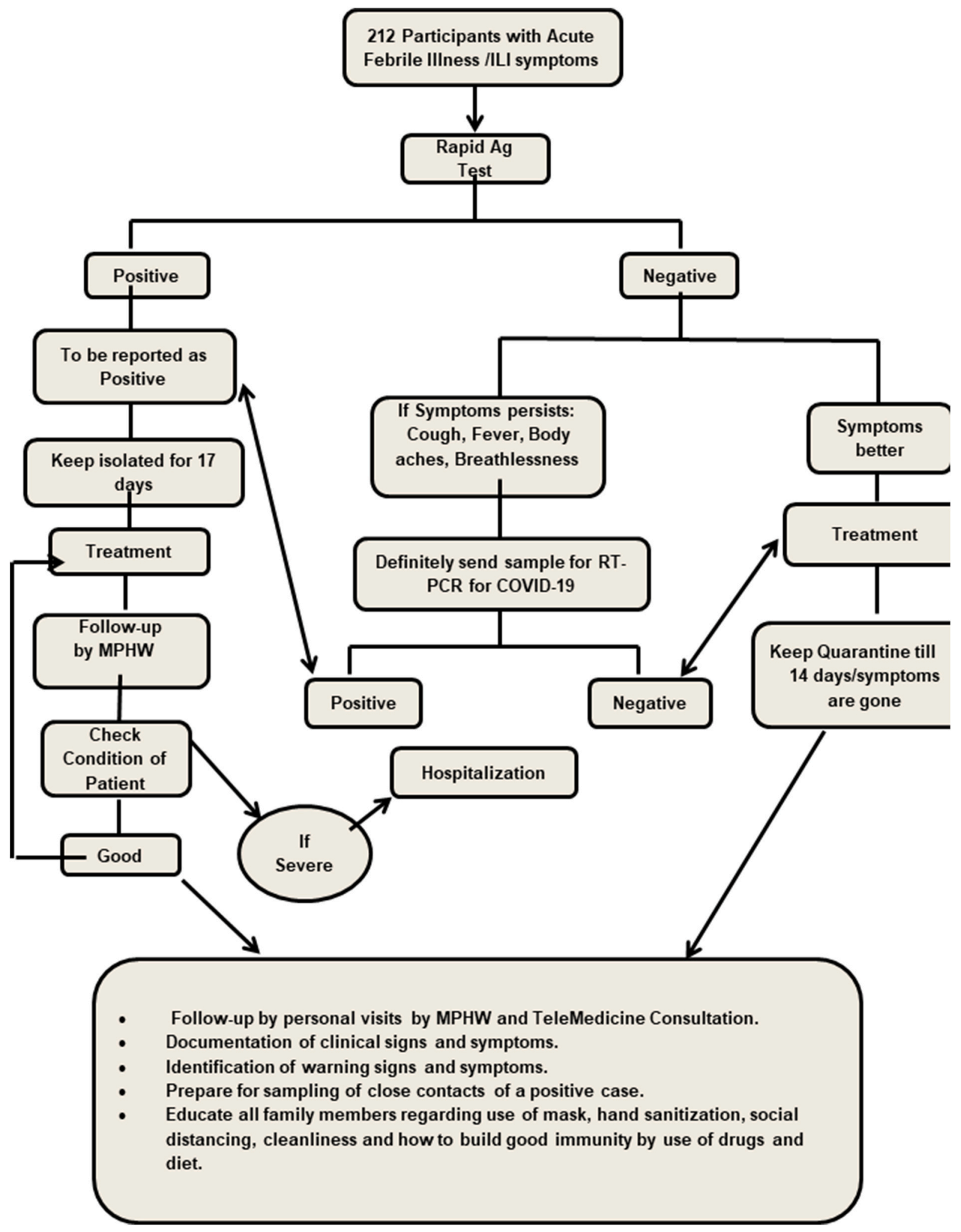

Fig. 4. The flowchart used for managing patients with Acute Febrile Illness/Influenza Like Infection (ILI) symptoms.

suspected cases should expand and aggressive contact tracing and isolation should continue. Hospitals, which have been radically reorganized to accommodate a surge of Covid-19 patients, should plan for severe dengue beds.
5. Conclusion

During the prevailing Covid-19 pandemic era, when the complete attention of healthcare professionals is on its intervention and prevention, there are high chances that co-occurrence of dengue in tropical countries during this time might go unnoticed due to overlapping 
symptoms of the two diseases. Based on the findings of the present study, biochemical markers such as vitamin D3, platelet count, and NLR represents potential targets for differential diagnosis of these two fatal viral diseases. More attention is needed in this direction by the health care professionals and policy makers to avoid mortality due to co-infection.

\section{Funding}

The authors did not receive support from any organization for the submitted work.

\section{Informed consent}

Informed consent was obtained from all individual participants included in the study.

\section{Consent to publish}

The participant has consented to the submission of the case report to the journal.

\section{Ethical approval}

Civil hospital Narwana, Jind, Government of Haryana, India. All procedures performed in studies involving human participants were in accordance with the ethical standards of the institutional research committee.

\section{Availability of data and material (data transparency)}

The datasets used and analysed during the current study are available from the corresponding author on reasonable request.

\section{Code availability (software application or custom code)}

Not Applicable.

\section{Authors' contributions}

HB conducted the tests and collected the data; VK and RM analysed the data and prepared the manuscript.

\section{Declaration of competing interest}

The authors have no conflicts of interest to declare that are relevant to the content of this article.

\section{Acknowledgments}

The support and research facilities provided by the Amity University Uttar Pradesh are acknowledged. Dr. Vijay Kumar, AUUP, is highly acknowledged for his scientific suggestions during preparation of manuscript. We also extend our acknowledgement to Dr Mohit Mittal, Medical Officer, Civil hospital, Narwana, Haryana, for his help during data collection and sample analysis.

\section{References}

1 Nacher M, Douine M, Gaillet M, et al. Simultaneous dengue and COVID-19 epidemics: difficult days ahead? PLoS Neglected Trop Dis. 2020;14(8), e0008426.

2 Saavedra-Velasco M, Chiara-Chilet C, Pichardo-Rodriguez R, Grandez-Urbina A, Inga-Berrospi F. Coinfection between dengue and covid-19: need for approach in endemic zones. Rev Fac Cien Med. 2020;77(1):52-54.

3 Masyeni S, Santoso MS, Widyaningsih PD, et al. Serological cross-reaction and coinfection of dengue and COVID-19 in Asia: experience from Indonesia. Int J Infect Dis. 2020;102:152-154.

4 Sarkar S, Khanna P, Singh AK. Impact of COVID-19 in patients with concurrent coinfections: a systematic review and meta-analyses. J Med Virol. 2021;93(4): 2385-2395.

5 Teotônio IMSN, de Carvalho JL, Castro LC, et al. Clinical and biochemical parameters of COVID-19 patients with prior or active dengue fever. Acta Trop. 2021;214:105782.

6 Cardona-Ospina JA, Arteaga-Livias K, Villamil-Gómez WE, et al. Dengue and COVID19, overlapping epidemics? An analysis from Colombia. J Med Virol. 2021;93(1): $522-527$.

7 Lorenz C, Azevedo TS, Chiaravalloti-Neto F. COVID-19 and dengue fever: a dangerous combination for the health system in Brazil. Trav Med Infect Dis. 2020;35, 101659.

8 Schmid MA, Gonzalez KN, Shah S, et al. Influenza and dengue virus co-infection impairs monocyte recruitment to the lung, increases dengue virus titers, and exacerbates pneumonia. Eur J Immunol. 2017;47(3):527-539.

9 Phadke R, Mohan A, Cavdaroğlu S, et al. Dengue amidst COVID-19 in India: the mystery of plummeting cases. J Med Virol. 2021;93(7), 4120.

10 Miah MA, Husna A. Coinfection, coepidemics of COVID-19, and dengue in dengueendemic countries: a serious health concern. J Med Virol. 2021;93(1):161-162.

11 Yan G, Lee CK, Lam LT, et al. Covert COVID-19 and false-positive dengue serology in Singapore. Lancet Infect Dis. 2020;20(5):536.

12 Zahedi M, Yousefi M, Abounoori M, et al. Liver Function in Novel Coronavirus Disease (COVID-19): A Systematic Review and Meta-Analysis. 2020. medRxiv.

13 Wang Q, Zhao H, Liu L-G, et al. Pattern of liver injury in adult patients with COVID19: a retrospective analysis of 105 patients. Military Medical Research. 2020;7:1-8.

14 Joshi JA, Puthiyedath R. Outcomes of ayurvedic care in a COVID-19 patient with hypoxia-A case report. J Ayurveda Integr Med. 2020. https://doi.org/10.1016/j. jaim.2020.10.006.

15 Yan G, Pang L, Cook AR, et al. Distinguishing Zika and dengue viruses through simple clinical assessment, Singapore. Emerg Infect Dis. 2018;24(8):1565.

16 Perdigão ACB, Ramalho ILC, Guedes MIF, et al. Coinfection with influenza A (H1N1) pdm09 and dengue virus in fatal cases. Mem Inst Oswaldo Cruz. 2016;111(9): 588-591.

17 Grant WB, Lahore H, McDonnell SL, et al. Evidence that vitamin D supplementation could reduce risk of influenza and COVID-19 infections and deaths. Nutrients. 2020; 12(4):988.

18 Martineau AR, Jolliffe DA, Hooper RL, et al. Vitamin D supplementation to prevent acute respiratory tract infections: systematic review and meta-analysis of individual participant data. BMJ. 2017:356.

19 Ebadi M, Montano-Loza AJ. Perspective: improving vitamin D status in the management of COVID-19. Eur J Clin Nutr. 2020;74(6):856-859.

20 Ilie PC, Stefanescu S, Smith L. The role of vitamin D in the prevention of coronavirus disease 2019 infection and mortality. Aging Clin Exp Res. 2020:1-4.

21 Lips P, Cashman KD, Lamberg-Allardt C, et al. Current vitamin D status in European and Middle East countries and strategies to prevent vitamin D deficiency: a position statement of the European Calcified Tissue Society. Eur J Endocrinol. 2019;180(4): P23-P54.

22 Gorji A, Ghadiri MK. The potential roles of micronutrient deficiency and immune system dysfunction in COVID-19 pandemic. Nutrition. 2020:111047.

23 Lagunas-Rangel FA. Neutrophil-to-lymphocyte ratio and lymphocyte-to-C-reactive protein ratio in patients with severe coronavirus disease 2019 (COVID-19): a metaanalysis. J Med Virol. 2020;92(10):1733-1734. https://doi.org/10.1002/jmv.25819. In this issue. 